\title{
Study Protocol for the Initial Choice of DPP-4 Inhibitor in Japanese Patients with Type 2 diabetes Mellitus: Effect of Linagliptin on QOL (INTEL-QOL) Trial
}

\author{
Tomoya Mita (D) - Toru Hiyoshi · Hidenori Yoshii · Hiroko Chimori • \\ Kazuo Ikeda $\cdot$ Junko Sato $\cdot$ Hirotaka Watada
}

Received: April 18, 2018 / Published online: May 9, 2018

(C) The Author(s) 2018

\section{ABSTRACT}

Introduction: Consideration of treatment-related quality of life (QOL) is important in diabetes management. However, no studies have compared the influence of dipeptidyl peptidase4 inhibitors versus metformin on treatment-

Enhanced digital features To view enhanced digital features for this article go to https://doi.org/10.6084/ m9.figshare.6200750.

T. Mita $(\bowtie) \cdot$ H. Watada

Department of Metabolism and Endocrinology, Juntendo University Graduate School of Medicine, Bunkyo-ku, Tokyo, Japan

e-mail: tom-m@juntendo.ac.jp

T. Hiyoshi

Division of Diabetes and Endocrinology, Japanese

Red Cross Medical Center, Shibuya-ku, Tokyo, Japan

H. Yoshii

Department of Medicine, Diabetology and Endocrinology, Juntendo Tokyo Koto Geriatric

Medical Center, Koto-ku, Tokyo, Japan

H. Chimori

Chimori Medical Clinic, Fukushima-ku, Osaka, Osaka, Japan

K. Ikeda

Ikeda Shinryojyo, Higashiosaka, Osaka, Japan

J. Sato

Department of Diabetes, Endocrinology and

Metabolism, Juntendo University Shizuoka

Hospital, Izunokuni, Shizuoka, Japan related QOL when used as first-line therapy in patients with type 2 diabetes mellitus.

Methods: This study is a prospective, randomized, open-label, multicenter, parallel-group, comparative study. Between June 2016 and December 2017, 44 participants who failed to achieve glycemic control despite diet and exercise therapy were recruited at 14 clinics and randomly allocated to linagliptin or metformin therapy. Treatment-related QOL was assessed with the Oral Hypoglycemic Agent Questionnaire, version 2 (OHA-Q ver. 2) and the selfadministered Diabetes Therapy-Related QOL (DTR-QOL) questionnaire. The primary study outcome is the difference in total OHA-Q ver. 2 score between the two treatment groups at the end of the study. The secondary outcomes include differences in the scores for each OHA$Q$ ver. 2 subscale between the two treatment groups at the end of the study, change in total DTR-QOL score and for each domain from baseline to the end of treatment, changes in glycemic control, and adverse events.

Planned outcome: The present study is designed to assess the effects of linagliptin on the treatment-related QOL. Results will be available in the near future. Study findings are expected to provide useful information on how to maintain or improve QOL in patients with type 2 diabetes mellitus treated with insulin.

Funding: Nippon Boehringer Ingelheim Co., Ltd. and Eli Lilly and Company.

Clinical trial registration: UMIN000022953. 
Keywords: Linagliptin;

Metformin; Questionnaire; Treatment-related quality of life; Type 2 diabetes mellitus

\section{INTRODUCTION}

One of the main objectives of diabetes management is to maintain quality of life (QOL) by preventing development or progression of complications [1]. In patients with type 2 diabetes mellitus (T2DM), advanced age, poor glycemic control, previous hypoglycemic episodes, and complex therapies are associated with lower QOL [2-5]. On the other hand, intensive frequency of follow-up was associated with improvements of QOL [6]. Given that decreased treatment-related QOL is associated with reduced patient motivation and adherence to treatment in patients with T2DM [7], consideration of treatment-related QOL is important when choosing oral anti-hypoglycemic agents (OHAs).

Treatment guidelines by the American Diabetes Association and European Association for the Study of Diabetes recommend metformin as first-line therapy when lifestyle modification alone fails to achieve or maintain optimal glycemic goals [8]. Metformin reduces blood glucose levels by improving insulin sensitivity, mainly in the liver. Metformin has an established evidence base showing that it is efficacious in lowering blood glucose levels without increasing the risk of hypoglycemia, with weight neutrality or loss. In addition, metformin is generally safe, well tolerated, and inexpensive [9]. Metformin might also reduce the incidence of cardiovascular events and improve survival [10]. However, a recent metaanalysis demonstrated that adherence to metformin was worse than for other OHAs such as sulfonylureas and thiazolidinediones [11]. In this regard, the most common adverse effects of metformin are gastrointestinal effects, such as nausea, vomiting, and diarrhea [12]. These symptoms might reduce patient adherence to metformin treatment. In addition, given that a sustained release preparation of metformin is not available in all countries, the need to take metformin more than twice daily may negatively affect patient adherence to therapy. These characteristics of metformin might reduce treatment-related QOL.

Conversely, the Japan Diabetes Society treatment guidelines recommend choosing suitable therapies in line with the dominant pathophysiological condition of each patient, such as insufficient insulin secretion or insulin resistance [13], because T2DM in East Asians is more strongly associated with beta cell dysfunction than insulin resistance and adiposity [14]. In this context, various types of OHAs, including metformin and dipeptidyl peptidase (DPP)-4 inhibitors, are chosen as first-line therapy in Japan [15].

DPP-4 inhibitors such as linagliptin enhance glucose-dependent insulin secretion from pancreatic beta cells via inhibiting the degradation of active incretins by DPP-4. In general, DPP-4 inhibitors are safe and well tolerated and do not cause weight gain [16]. Sitagliptin was shown to improve QOL in a single arm-study [17]. Owing to these characteristics, DPP-4 inhibitors are now the most frequently prescribed first-line agents for T2DM in Japan [18].

In this study, to investigate how these treatment regimens affect QOL of patients with T2DM, we evaluated the effect of linagliptin versus metformin on treatment-related $\mathrm{QOL}$ using the Oral Hypoglycemic Agent Questionnaire, version 2 (OHA-Q ver. 2) and the selfadministered Diabetes Therapy-Related QOL (DTR-QOL) questionnaire [19]. Although these questionnaires are newly developed, they are valid $[19,20]$.

\section{MATERIALS AND METHODS}

\section{Study Design}

The Initial choice of DPP-4 inhibitor in Japanese T2DM patients: Effect of Linagliptin on QOL (INTEL-QOL) study is a prospective, randomized, open-label, multicenter, parallel-group, comparative trial. This study is planned solely to evaluate the QOL. This study has been registered on the University Hospital Medical Information Network Clinical Trials Registry (UMIN-CTR), a non-profit organization in Japan 
that meets the requirements of the International Committee of Medical Journal Editors (ICMJE) (UMINO00022953).

\section{Study Population}

Japanese patients with T2DM who regularly attended the outpatient diabetes clinics of 14 institutions (Chimori Clinic, Ikeda Clinic, Yamamoto Clinic, Japanese Red Cross Medical Center, Juntendo Tokyo Koto Geriatric Medical Center (Department of Medicine, Diabetology and Endocrinology), Juntendo University Graduate School of Medicine (Department of Metabolism and Endocrinology), Juntendo University Shizuoka Hospital (Department of Diabetes, Endocrinology, and Metabolism), Sawaki Internal Medicine and Diabetes Clinic, Shimizu Clinic, Tanaka Clinic, Menju Clinic, Misaki Naika Clinic, Musashino Family Clinic, and Yasuda Clinic) in Japan were asked to participate in this study. The inclusion criteria are as follows: (1) T2DM not at target blood glucose control as specified in the Treatment Guide for Diabetes by the Japan Diabetes Society [13] despite the introduction of diet and/or exercise therapy; (2) patients who are considered to newly initiate OHAs in addition to dietary and/ or exercise therapy; (3) age $\geq 20$ years and $<75$ years, regardless of gender; and (4) written informed consent for study participation. The following exclusion criteria are used: (1) type 1 or secondary diabetes; (2) acute diabetic complications within the past 6 months; (3) congestive heart failure or myocardial infarction requiring pharmacotherapy; (4) unstable angina pectoris or coronary bypass surgery within the past 6 months; (5) chronic cirrhosis or chronic active hepatitis; (6) artificial dialysis or moderate renal dysfunction; (7) aspartate aminotransferase or alanine aminotransferase levels more than three times higher than the upper limit of the normal range; (8) direct bilirubin more than three times higher than the upper limit of the normal range, clinically abnormal thyroid-stimulating hormone level, or fasting triglyceride levels $>7.9 \mathrm{mmol} /$ $\mathrm{L}$; (9) treatment with any type of antidiabetic drug; (10) dementia, possible dementia, or mental disorder; (11) insufficient judgment ability or illiteracy; (12) requiring consent for study participation from a legal representative; (13) pregnancy, lactation, possibly pregnancy, or planning to become pregnant during the study period; (14) history of hypersensitivity to investigational drugs; (15) present or past history of a malignant tumor, unless there was no current medical therapy, no recurrence to date, and no risk of recurrence during this study; and (16) judged as ineligible by the clinical investigators.

The subjects were screened consecutively. Patients that met the above eligibility criteria were asked to participate in the present study. All patients who agreed to participate were enrolled in the study. The protocol has been approved by the institutional review board of each participating institution in compliance with the Declaration of Helsinki and current legal regulations in Japan. All procedures followed were in accordance with the ethical standards of the responsible committee on human experimentation (institutional and national) and with the 1964 Declaration of Helsinki, as revised in 2013. Informed consent was obtained from all patients for being included in the study.

\section{Randomization and Study Intervention}

Patients were registered at the INTEL-QOL trial's administration office via the internet. Once enrolled, patients were randomly assigned to either the linagliptin group or the metformin group. Randomization was performed using a dynamic allocation method based on age $(<65$ or $\geq 65$ years) and gender (male or female).

Patients in the linagliptin group were started on linagliptin $5 \mathrm{mg}$ once daily. Patients in the metformin group were started on metformin $500 \mathrm{mg}$ twice daily. The dose of metformin was increased to a maximum dose of $2250 \mathrm{mg}$ once daily when HbA1c was $\geq 7.0 \%$ [13]. The addition of OHAs other than the study drugs and insulin is not permitted in both groups during the study. 


\section{Study Variables and Schedule}

The study variables and schedule are shown in Table 1 and Fig. 1. The study period consists of 24 weeks after registration (registration period, June 2016 to December 2017; full study duration, June 2016 to September 2018). All randomized participants will be followed until the end of the scheduled study, regardless of adherence to or discontinuation of the study medication for any reason. Clinical outcome, adherence, and adverse events will be ascertained. Clinical and biochemical data are collected at baseline and 24 weeks after randomization. Blood samples are obtained after overnight fasting at baseline and 24 weeks after randomization. Urinary albumin excretion is measured by latex agglutination assay using a spot urine sample at baseline and 24 weeks after randomization. In addition, participants were asked to record usage of their study drug on a medication record during the study period.

\section{STUDY OUTCOMES}

The primary study outcome is the difference in the total OHA-Q ver. 2 score between the two treatment groups at the end of the study. The secondary outcomes are (1) the difference in score for each OHA-Q ver. 2 subscale between the two treatment groups at the end of the study; (2) change in total DTR-QOL score and score for each DTR-QOL domain from baseline to 24 weeks; (3) change in $\mathrm{HbA1c}$ from baseline to 24 weeks; (4) differences in the proportion of patients who achieved HbA1c $<6.0 \%$ or $<7.0 \%$ at the end of the study or whose HbA1c levels improved by more than $0.5 \%$ from baseline to 24 weeks; (5) change in fasting glucose levels from baseline to 24 weeks; (6) differences in the proportion of patients who achieved HbA1c $<$ $7.0 \%$ and had an improved total DTR-QOL score at the end of the study; (7) differences in the prevalence of hypoglycemic events; (8) differences in the proportion of gastrointestinal symptoms such as nausea, vomiting, and

Table 1 Variables and data collection schedule

\begin{tabular}{|c|c|c|c|c|}
\hline & \multirow[t]{2}{*}{ Registration } & \multicolumn{3}{|c|}{ Treatment period } \\
\hline & & Baseline & Baseline to week 24 & Week 24 \\
\hline Patient characteristics & $\bigcirc$ & & & \\
\hline Body weight & & O & & $\bigcirc$ \\
\hline Blood pressure & & O & & O \\
\hline Blood chemistry $^{a}$ & & $\bigcirc$ & & $\bigcirc$ \\
\hline Urinary albumin excretion & & $\bigcirc$ & & O \\
\hline Oral Hypoglycemic Agent Questionnaire, version 2 & & & & $\bigcirc$ \\
\hline Diabetes Therapy-Related Quality of Life questionnaire & & $\bigcirc$ & & $\bigcirc$ \\
\hline Hypoglycemic events & & O & $\bigcirc$ & $\bigcirc$ \\
\hline Gastrointestinal events & & O & $\bigcirc$ & $\bigcirc$ \\
\hline Other adverse events & & ○ & $\bigcirc$ & $\bigcirc$ \\
\hline Adherence & & $\bigcirc$ & $\bigcirc$ & $\bigcirc$ \\
\hline
\end{tabular}

${ }^{a}$ Includes aspartate aminotransferase, alanine aminotransferase, $\gamma$-glutamyl transpeptidase, total bilirubin, blood urea nitrogen, creatinine, estimated glomerular filtration rate, uric acid, total cholesterol, high-density lipoprotein cholesterol, low-density lipoprotein cholesterol, triglycerides, fasting plasma glucose, fasting inulin, HbAlc, amylase, and thyroidstimulating hormone 


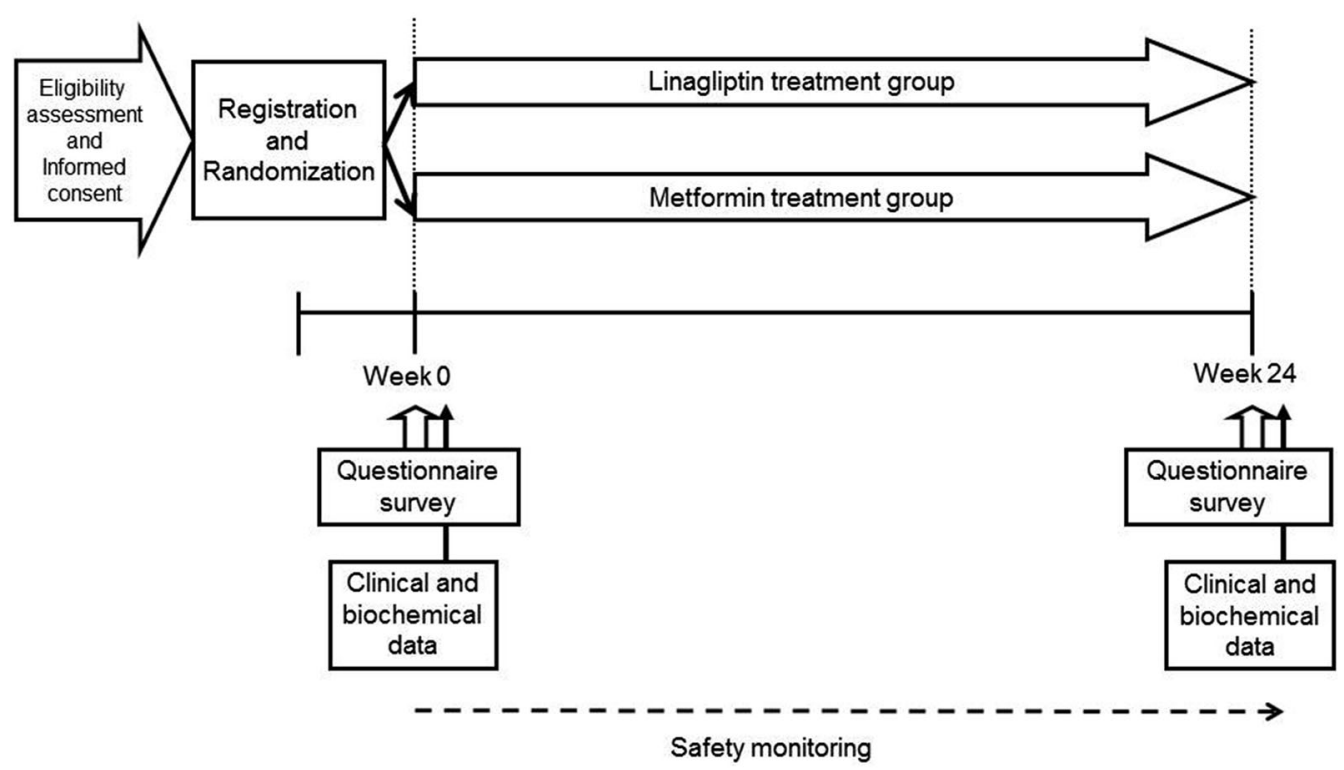

Fig. 1 Flow chart of the study schedule

diarrhea; (9) differences in the proportion of patients with hospitalization for heart failure; and (10) differences in the proportion of other adverse effects.

\section{OHA-Q ver. 2}

The OHA-Q ver. 2 is a 23 -item, self-administered assessment with three subscales including treatment convenience (9 items; questions 1-9), somatic symptoms (8 items; questions 11-21), and satisfaction (3 items; questions 10, 22, and 23) (Table 2) [20]. The response to each question is scored using a four-point Likert-type scale that ranged from 1 to 4 points. Answers are converted to scores of $0-3$. Subscale scores are calculated by summing the response to the items in each subscale. Higher scores represent higher satisfaction.

\section{DTR-QOL Questionnaire}

The DTR-QOL is a 29-item, self-administered assessment with four primary scales including burden on social activities and daily activities
(13 items), anxiety and dissatisfaction with treatment (8 items), hypoglycemia (4 items), and satisfaction with treatment (4 items) (Table 3) [19]. The DTR-QOL can evaluate the effect of diabetes treatment on patient QOL with high reliability and validity [19]. The response to each question is scored using a seven-point Likert-type scale that ranges from 1 (strongly agree) to 7 (strongly disagree). The scale for items $26-29$ is reversed, so that 7 represent the highest QOL score. Domain scores are calculated by summing the response to the items in each domain. Scores are then converted to a range of $0-100$. Higher scores represent higher QOL. In each domain, average scores are calculated.

\section{Sample Size}

The sample size was not calculated on the basis of scientific evidence because this was an exploratory study. Assuming a treatment dropout rate of $10 \%$, the target number of enrolled patients was set at 22 subjects per group, or 44 subjects in total. 
Table 2 Oral Hypoglycemic Agent Questionnaire, version 2 questionnaire and subscale structure

Q1. Do you ever forget to take your diabetes medication? (How many times a week?)

Q2. Are you concerned about the size of the tablets, difficulty swallowing the tablets, etc., when taking diabetes medication?

Q3. Is handling/carrying/preparing to taking diabetes medicine troublesome?

Q4. Are you concerned about being seen by others when taking diabetes medication outside of your home?

Q5. Is it a burden to eat meals at regular times in order to take diabetes medication?

Q6. Is being punctual in taking your diabetes medication and your meals troublesome?

Q7. Is it a burden to take diabetes medication at predetermined times?

Q8. Is the dosing frequency for diabetes medication a hassle?

Q9. Is it difficult to take diabetes medication outside of your home?

Q10. Do you want to continue to take your current diabetes medication?

Q11. Are you concerned about passing gas or rumbling in your stomach?

Q12. Are you concerned about diarrhea?

Q13. Are you concerned about constipation?

Q14. Are you concerned about weight gain?

Q15. Are you concerned about readily becoming hungry?

Q16. Are you concerned about having an upset stomach?

Q17. Are you concerned about swelling of your body?

Q18. Are you worried about hypoglycemia?

Q19. Are you concerned about frequent urination?

Q20. Are you concerned about thirsty?

Q21. Are you concerned about discomfort with urination or genital pruritus?

Q22. Are you satisfied with your current blood glucose control?

Q23. Are you satisfied with your current treatment with the diabetes medication?

The question numbers in the Oral Hypoglycemic Agent Questionnaire, version 2 questionnaire are used in this table

\section{Safety Evaluation}

All adverse effects (AEs) during the study are documented to ensure patient safety. AEs are defined as any untoward medical occurrence in a clinical trial subject who has received a medicinal product. AEs do not necessarily have a causal relationship with treatment. The association between AEs and the study medication is classified as either being associated with or not associated with the study drug by one of the investigators. All associated AEs that result in the withdrawal of a subject from the study are monitored until resolution. Serious AEs are defined as death or life-threatening events that required hospitalization, prolonged existing hospitalization, or resulted in persistent or significant disability or incapacity that requires intervention to prevent permanent impairment or damage. 
Table 3 Diabetes Therapy-Related QOL questionnaire and domain structure

Domain 1: Burden on social activities and daily activities

Q1. My current diabetes treatment interferes with my work and activities

Q2. My current diabetes treatment limits the scope of my activities

Q3. It is difficult to find places on time for my current diabetes treatment

Q4. My current diabetes treatment interferes with group activities and personal friendships

Q5. It is a burden getting up at a certain time every morning for my current diabetes treatment

Q6. With my current diabetes treatment, the restricted meal times are a burden

Q7. When I eat out, it is difficult to manage my current diabetes treatment

Q8. I feel like my current diabetes treatment takes away the enjoyment of eating

Q9. With my current diabetes treatment, it is hard to curb my appetite

Q10. The time and effort to manage my current diabetes treatment are a burden

Q11. I am constantly concerned about time to manage my current diabetes treatment

Q12. Pain due to my current diabetes treatment is uncomfortable

Q13. Gastrointestinal symptoms (nausea, passing gas, diarrhea, abdominal pain) due to my current diabetes treatment are uncomfortable

Domain 2: Anxiety and dissatisfaction with treatment

Q14. I am bothered by weight gain with my current diabetes treatment

Q19. I have uncomfortable symptoms due to hyperglycemia (high blood glucose)

Q20. I am worried about high blood glucose

Q21. I am dissatisfied that my blood glucose is unstable (high and low)

Q22. I am worried that complications might worse with my current diabetes treatment

Q23. I got anxious thinking about living while on my current diabetes treatment

Q24. I find it unbearable to think that even if I continue my current diabetes treatment, my diabetes may not be cured

Q25. I am concerned that if I continue my current diabetes treatment, the efficacy (effectiveness) may diminish

Domain 3: Hypoglycemia

Q15. I worry about low blood glucose due to my current diabetes treatment

Q16. I am scared because of low blood glucose

Q17. I am sometimes bothered by low blood glucose

Q18. Symptoms due to low blood glucose are uncomfortable

Domain 4: Satisfaction with treatment

Q26. Overall, I am satisfied with my current blood sugar control (glycemic control)

Q27. With my current diabetes treatment, I am confident that I can maintain good blood glucose control

Q28. I am hopeful about the future with my current diabetes treatment

Q29. With regard to diabetes treatment, I am satisfied with current treatment methods

The question numbers in the Diabetes Therapy-Related-QOL questionnaire are used in this table 


\section{Statistical Analysis}

Efficacy will be analyzed using the full analysis data set, except for the safety analysis. To compare the total OHA-Q ver. 2 score and each OHA-Q ver. 2 domain score between the two treatment groups at the end of the study, statistical analysis will include Student's $t$ test and analysis of covariance (ANCOVA) models that include treatment group as a fixed effect and the allocation factors of age ( $<65$ or $\geq 65$ years) and gender (male or female) as covariates. To compare the changes in total and domain DTRQOL scores, HbA1c, and fasting blood glucose between the two treatment groups at the end of the study, statistical analysis will include Student's $t$ test and ANCOVA models that include treatment group as fixed effects and (1) each baseline value as a covariate, (2) allocation factors as covariates, and (3) each baseline value and allocation factors as covariates. For the other variables, comparisons between the groups will be assessed using Student's $t$ test or the Wilcoxon rank-sum test for continuous variables, and the chi-square test or Fisher's exact test for categorical variables. Changes from baseline to week 24 within a treatment group will be assessed with the one-sample $t$ test or Wilcoxon signed-rank test. The correlation between total OHA-Q ver. 2 score at the end of study or change in DTR-QOL score from baseline to 24 weeks will be evaluated using Pearson's and Spearman's correlation coefficients. All statistical tests will be two-sided with a significance level of 5\%. All analyses will be performed using SAS software, version 9.4 (SAS Institute, Cary, NC). The statistical analysis plan will be written by an independent statistician.

\section{Strengths and Limitations of Study Protocol}

The strength is a prospective, randomized, open-label, multicenter, parallel-group, comparative trial design. In addition, two questionnaires for evaluating QOL used in this study are valid. On the other hand, the limitations are the small number of participants and the openlabel, exploratory trial design.

\section{ACKNOWLEDGMENTS}

The authors wish to thank the study investigators for their contributions to this study and the participants of study.

Funding. This study was financially supported by Nippon Boehringer Ingelheim Co., Ltd. and Eli Lilly and Company. The article processing charges and editorial assistance were funded by the authors. All authors have full access to all of the data in this study and take complete responsibility for the integrity of the data and accuracy of the data analysis.

Editorial Assistance. The authors thank all the clinical staff for their assistance with the execution of the clinical trial, and Soiken Inc. for their technical assistance in the launch and execution of this trial.

Authorship. All named authors meet the International Committee of Medical Journal Editors (ICMJE) criteria for authorship for this article, take responsibility for the integrity of the work as a whole, and have given their approval for this version to be published.

Disclosures. Tomoya Mita has received research funds from MSD K.K. and Takeda Pharmaceutical Co., Ltd., Kowa Pharmaceutical Co., Ltd., Sanwa Kagaku Kenkyusho Co., Ltd., Nippon Boehringer Ingelheim Co., Ltd., Mitsubishi Tanabe Pharma Co., and Ono Pharmaceutical Co., Ltd. and lecture fees from AstraZeneca K.K., Nippon Boehringer Ingelheim Co., Ltd., Eli Lilly and Company, Kowa Pharmaceutical Co., Ltd., Mitsubishi Tanabe Pharma Co., MSD K.K., Ono Pharmaceutical Co., Ltd., Novo Nordisk Pharma Ltd., and Takeda Pharmaceutical Co., Ltd. Junko Sato has received research funds from research funds from MSD K.K. and Takeda Pharmaceutical Co., Ltd., Kowa Pharmaceutical Co., Ltd., Sanwa Kagaku Kenkyusho Co., Ltd., Nippon Boehringer Ingelheim Co., Ltd., Mitsubishi Tanabe Pharma Co., and Ono Pharmaceutical Co., Ltd. Hirotaka Watada has received honoraria for scientific lectures from MSD K.K., Eli Lilly and Company, Takeda Pharmaceutical Co., Ltd., Novartis Pharma K.K., 
Sumitomo Dainippon Pharma Co., Ltd., Sanofi K.K., and Daiichi Sankyo Co., Ltd., and research funds from MSD K.K., Eli Lilly and Company, Takeda Pharmaceutical Co., Ltd., Kowa Pharmaceutical Co., Ltd., Mochida Pharmaceutical Co., Ltd., Sanwa Kagaku Kenkyusho Co., Ltd., Novo Nordisk Pharma Ltd., Kissei Pharmaceutical Co., Ltd., Novartis Pharma K.K., Nippon Boehringer Ingelheim Co., Ltd., AstraZeneca K.K., Astellas Pharma Inc., Mitsubishi Tanabe Pharma Co., Sumitomo Dainippon Pharma Co., Ltd., Abbott Japan Co., Ltd., Sanofi K.K., Pfizer Japan Inc., and Daiichi Sankyo Co., Ltd. Toru Hiyoshi, Hidenori Yoshii, Hiroko Chimori, and Kazuo Ikeda have nothing to disclose.

Compliance with Ethics Guidelines. The protocol has been approved by the institutional review board of each participating institution in compliance with the Declaration of Helsinki and current legal regulations in Japan. All procedures followed were in accordance with the ethical standards of the responsible committee on human experimentation (institutional and national) and with the Helsinki Declaration of 1964 , as revised in 2013. Informed consent was obtained from all patients for being included in the study.

Data Availability. The analyzed data sets are available from the corresponding author on reasonable request.

Open Access. This article is distributed under the terms of the Creative Commons Attribution-NonCommercial 4.0 International License (http://creativecommons.org/licenses/ by-nc/4.0/), which permits any noncommercial use, distribution, and reproduction in any medium, provided you give appropriate credit to the original author(s) and the source, provide a link to the Creative Commons license, and indicate if changes were made.

\section{REFERENCES}

1. Araki E, Haneda M, Kasuga M, et al. New glycemic targets for patients with diabetes from the Japan
Diabetes Society. J Diabetes investig. 2017;8(1):123-5.

2. Papazafiropoulou AK, Bakomitrou F, Trikallinou A, et al. Diabetes-dependent quality of life (ADDQOL) and affecting factors in patients with diabetes mellitus type 2 in Greece. BMC Res Notes. 2015;8:786.

3. Yfantopoulos J, Hatzikou M, Rombopoulos G, Panitti E, Latsou D. The prevalence of hypoglycemia and its impact on the quality of life of type 2 diabetes mellitus patients in Greece (the Hypo study). Value Health. 2014;17(7):A356.

4. Depablos-Velasco P, Salguero-Chaves E, Mata-Poyo J, Derivas-Otero B, Garcia-Sanchez R, Viguera-Ester P. Quality of life and satisfaction with treatment in subjects with type 2 diabetes: results in Spain of the PANORAMA study. Endocrinol Nutr. 2014;61(1):18-26.

5. Imayama I, Plotnikoff RC, Courneya KS, Johnson JA. Determinants of quality of life in adults with type 1 and type 2 diabetes. Health Qual Life Outcomes. 2011;9:115.

6. Hu M, Zhou Z, Zeng F, Sun Z. Effects of frequency of follow-up on quality of life of type 2 diabetes patients on oral hypoglycemics. Diabetes Technol Ther. 2012;14(9):777-82.

7. Ishii $\mathrm{H}$, Anderson JH Jr, Yamamura A, Takeuchi M, Ikeda I. Improvement of glycemic control and quality-of-life by insulin lispro therapy: assessing benefits by ITR-QOL questionnaires. Diabetes Res Clin Pract. 2008;81(2):169-78.

8. Inzucchi SE, Bergenstal RM, Buse JB, et al. Management of hyperglycemia in type 2 diabetes, 2015: a patient-centered approach: update to a position statement of the American Diabetes Association and the European Association for the Study of Diabetes. Diabetes Care. 2015;38(1):140-9.

9. Holman RR, Paul SK, Bethel MA, Matthews DR, Neil HA. 10-year follow-up of intensive glucose control in type 2 diabetes. $\mathrm{N}$ Engl $\mathrm{J}$ Med. 2008;359(15):1577-89.

10. UK Prospective Diabetes Study (UKPDS) Group. Effect of intensive blood-glucose control with metformin on complications in overweight patients with type 2 diabetes (UKPDS 34). Lancet. 1998;352(9131):854-65.

11. McGovern A, Tippu Z, Hinton W, Munro N, Whyte $M$, de Lusignan $S$. Comparison of medication adherence and persistence in type 2 diabetes: a systematic review and meta-analysis. Diabetes Obes Metab. 2018;20(4):1040-3. 
12. Kirpichnikov D, McFarlane SI, Sowers JR. Metformin: an update. Ann Intern Med. 2002;137(1):25-33.

13. Japan Diabetes Society. Treatment guide for diabetes. Tokyo: Bunkodo; 2010. p. 1-118.

14. Kodama K, Tojjar D, Yamada S, Toda K, Patel CJ, Butte AJ. Ethnic differences in the relationship between insulin sensitivity and insulin response: a systematic review and meta-analysis. Diabetes Care. 2013;36(6):1789-96.

15. Oishi M, Yamazaki K, Okuguchi F, et al. Changes in oral antidiabetic prescriptions and improved glycemic control during the years 2002-2011 in Japan (JDDM32). J Diabetes Investig. 2014;5(5):581-7.

16. Ohmura H, Mita T, Taneda Y, et al. Efficacy and safety of sitagliptin in Japanese patients with type 2 diabetes. J Clin Med Res. 2015;7(4):211-9.
17. Sakamoto Y, Oyama J, Ikeda $\mathrm{H}$, et al. Effects of sitagliptin beyond glycemic control: focus on quality of life. Cardiovasc Diabetol. 2013;12:35.

18. Seino Y, Kuwata H, Yabe D. Incretin-based drugs for type 2 diabetes: focus on East Asian perspectives. J Diabetes Investig. 2016;7(Suppl 1):102-9.

19. Ishii H. Development and psychometric validation of the Diabetes Therapy-Related QOL (DTR-QOL) questionnaire. J Med Econ. 2012;15(3):556-63.

20. Nakajima H, Okada S, Mohri T, et al. Dapagliflozin improves treatment satisfaction in overweight patients with type 2 diabetes mellitus: a patient reported outcome study (PRO study). Diabetol Metab Syndr. 2018;10:11. 